\title{
Effect of Doped Insulating Nanoparticles on the Electro-Optical Characteristics of Nematic Liquid Crystals
}

\author{
Wei-Ting Chen, Pei-Shiang Chen, and Chih-Yu Chao* \\ Department of Physics, National Taiwan University, Taipei 10617, Taiwan \\ Received August 1, 2008; accepted October 9, 2008; published online January 20, 2009
}

In this paper, the transient currents and the voltage holding ratio (VHR) generated by an applied direct voltage in nematic liquid crystal (LC) cells are measured. The experimental results show that doping insulating nanoparticles such as $\mathrm{ZnO}, \mathrm{TiO}_{2}$, and $\mathrm{Si}_{3} \mathrm{~N}_{4}$ in nematic $\mathrm{LCs}$ leads to a reduction in the moving-ion density in LC cells and drastically reduces the transient currents as well as threshold voltage. This doping method improves the electro-optical characteristics in nematic LC cells. Moreover, we found that the VHR values maintained higher than $95 \%$ in these doped LC cells. Such a high VHR observed in insulating nanoparticle-doped LC cells is a superior feature for LC display applications. (C) 2009 The Japan Society of Applied Physics

\section{Introduction}

Presenting information is essential in many aspects of our daily life. Among all display systems, liquid crystal display (LCD) occupies more than half of the display market as a whole on account of many advantages, including the flat panel, small weight, high definition, small driving voltages, and low power consumption. Currently, almost all LCDs are manufactured with nematic liquid crystal (LC) mixtures. And one of the most common modes for nematic LC displays is twisted nematic (TN) mode. Although the nematic LCs are the dominant materials in display market and for switchable optical devices, the degrading tendency of the performance is undoubtedly a significant barrier for practical applications. The performance of a LC device is mainly affected by the alignment layers and the LC mixtures used. One of the most crucial causes of performance degradation for nearly all the LCDs is the flowing ions within the LC devices. These ions may come from the LCs, the alignment layers, the surrounding glue, be generated by ultraviolet polymerization, or be produced during the filling process. When a voltage is applied across the LC cell, such ions begin to move and finally are adsorbed by the alignment layers. The adsorbed ionic layers, called the electric double layers, ${ }^{1)}$ create an inner direct current field and decrease the effective voltage of the LC layers. This screening effect increases the threshold voltage required for the display and gives rise to flicking as well as image sticking. In order to perform high-quality images, the LCs employed in a LCD must possess a sufficiently high resistivity and a reasonably low concentration of ion impurity. It is the reason why cyano compounds such as E7 and 5CB, known to have high conductivity and ion trapping near the alignment layers, are not used in the LCD industry.

Recently, considerable attention has been paid to the influence of ions on the electro-optical characteristics of LCDs. Some experiments have been done to explain the motion of charges in the LC cells, such as the studies on the transient currents, ${ }^{2)}$ measurements of the electro-optical properties, ${ }^{3)}$ influence of ion transport on LC switching, ${ }^{4)}$ and the simulation of the voltage holding ratio. ${ }^{5)}$ However, the voltage holding ratio (VHR) of the LC cells was not directly measured in the experiments above. Nevertheless,

*E-mail address: cychao@phys.ntu.edu.tw the VHR is an important performance parameter for an active matrix addressed LCD. Each pixel in the LCD is driven by a thin-film transistor charging the pixels by signal pulses. The storing charges of a pixel should be maintained until the pixel is addressed in the next driving frame. If the capacity of keeping the charge at a pixel is insufficient, a decrease in contrast, non-uniformity of the brightness or even image flickering can occur. The capability of keeping charges is called the VHR defined as the ratio of voltage at a pixel at the end of the frame time to the applied pulse voltage. For a high-end LCD, the VHR must be high and uniform.

To improve the electro-optical behavior associated with the ion impurity, at least two primary approaches have been made. One is to modify the surface of LC cells by coating an ion trapping layer above the indium tin oxide (ITO) electrode $^{6)}$ and the other is the structure modification of the LC host by incorporation with nanoscale materials. ${ }^{7,8)}$ There are many researches about the electro-optical characteristic of the LC-nanoparticles mixtures in recent years because the method to modify LC by doping nanoparticles in the LC is much easier than the conventional chemical synthetic method. The modification of the characteristic of the LCs will be determined by the type of the nanoparticles used as guest dopants. To date, there are four main kinds of nanoparticles doped in LC materials, such as conductive nanoparticles, ${ }^{9,10)}$ inorganic nanoparticles, ${ }^{11)}$ ferroelectric nanoparticles, ${ }^{12,13)}$ and carbon nanotubes (CNTs). The doped LCs exhibit many special characteristic, such as frequency modulation, enhancement of the dielectric anisotropy and birefringence, reduced threshold voltage and induced vertical alignment. CNTs have been used frequently so far as guest dopants in nematic LCs owing to many of their unique physical, chemical and electronic properties. Some advantages were found in the CNT-doped LC cells such as the reduction of residual $\mathrm{dc},{ }^{7)}$ which is related to the image sticking problems. But in our previous work, ${ }^{14)}$ we found that the CNT-doped cells will possess a very low VHR value when the applied voltage is above the Freedericksz threshold. This phenomenon is due to the alignment of the CNTs along the LC director and the CNT's anisotropic conductivity, which is much larger along its long axis than perpendicular to it. ${ }^{15)}$

In this paper, we dope the insulating nanoparticles such as $\mathrm{ZnO}, \mathrm{TiO}_{2}$, and $\mathrm{Si}_{3} \mathrm{~N}_{4}$ in nematic LC cells. Our experimental 


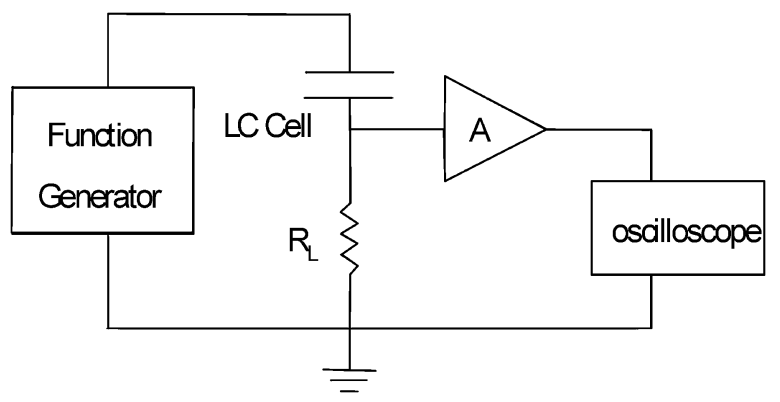

Fig. 1. Schematic illustration of the setup for the transient current measurement.

results show that the addition of insulating nanoparticles leads to a reduction of the transported ion concentration and the threshold voltage. Simultaneously, a high VHR value is observed.

\section{Experimental Methods}

For cell fabrication, the nematic LC MJ9915 offered by Chung-Hwa Picture Tube was used. And the insulating nanoparticles are purchased from Nanostructured \& Amorphous Materials. In this work, we use three kinds of insulating nanoparticles $\mathrm{Si}_{3} \mathrm{~N}_{4}, \mathrm{ZnO}$, and $\mathrm{TiO}_{2}$ as guest dopants with diameters about 25-35 nm. Each empty LC cell was manufactured with two flat glass substrates coated with ITO electrodes and polyimide alignment layers for planar orientation in a $90^{\circ} \mathrm{TN}$ configuration, where a cell gap of $4 \mu \mathrm{m}$ was ensured by spacers. The overlapped area of the electrodes was $1 \mathrm{~cm}^{2}$. The host-guest LC-nanoparticles mixtures were prepared by adding nanoparticles into LC MJ9915 in its isotropic phase. The mixtures were ultrasonicated and then stirred to promote dispersion. The mixtures with different kinds of nanoparticles and concentration were then introduced into empty cells by capillary action in the isotropic phase. A schematic illustration of the transient current measurement is shown in Fig. 1. For the measurements we apply a square voltage with amplitude $1.5 \mathrm{~V}_{\mathrm{pp}}$ and frequency $0.5 \mathrm{~Hz}$. The resulting leakage current is amplified and visualized on the scope. We repeat the waveforms several times to lower the noise by software averaging. To exclude the charging current caused by the reorientation of the LC molecules, we use voltages below the Freedericksz threshold. Because this charging current interferes with the ion current, voltages above threshold would destroy the reliability of the transient current measurements. ${ }^{16)}$ Transmittance of the TN LC cell from a $\mathrm{He}-\mathrm{Ne}$ laser was also measured with a photo detector. All cells were measured by using a cross-polarizer configuration, yielding normally white (NW) mode, under an applied triangle wave with frequency $0.02 \mathrm{~Hz}$ and amplitude $5 \mathrm{~V}$. The VHR values were measured using the VHR measurement system (Toyo). The detailed experimental method for VHR measurement can be found in ref. 5 .

\section{Results and Discussion}

The transient current of the doped LC cells induced by the polarity-reversed voltage is compared with the undoped one (see Fig. 2). There are three main characteristics in the transient current. One has known that the capacitive

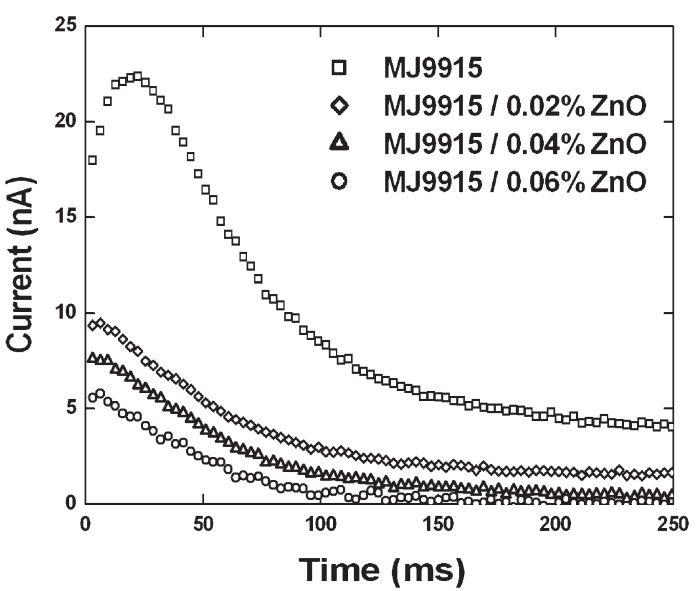

(a)

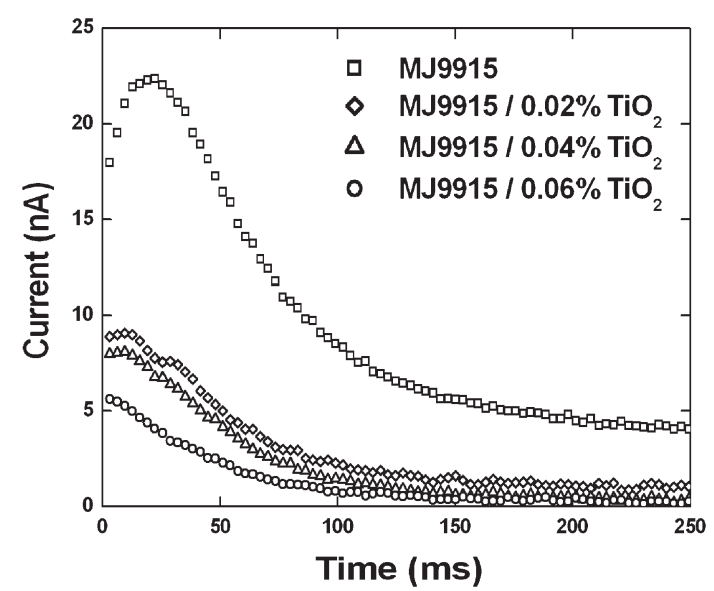

(b)

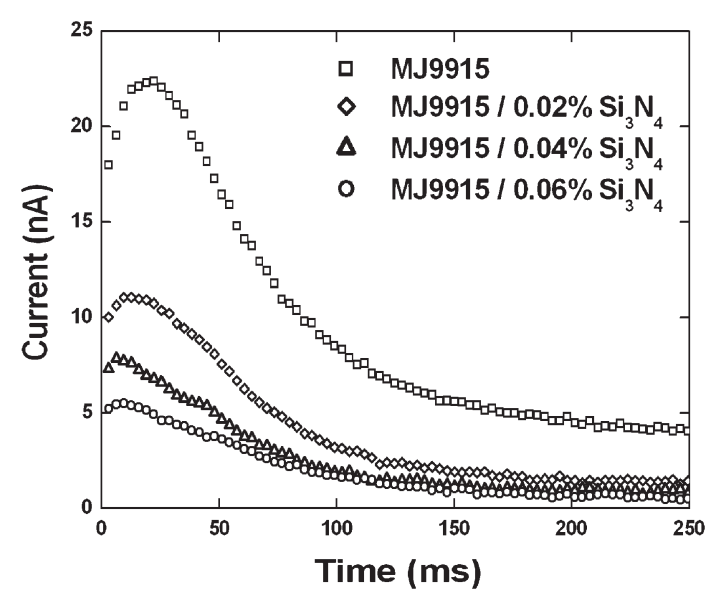

(c)

Fig. 2. Measured transient currents of (a) $\mathrm{ZnO}$, (b) $\mathrm{TiO}_{2}$, and (c) $\mathrm{Si}_{3} \mathrm{~N}_{4}$ doped LC cells.

charging current appears within a very short time after the polarity is reversed, followed by a transient current peak caused by the moving ions. If the applied voltage is above the Freedericksz threshold, the third kind of the current caused by the reorientation of the LC molecules will be observed. Since the aim of this paper is focused on the moving ion current, we use the voltage below the Freedericksz threshold to avoid the interference between the ion current and the LC reorientation current. The reason for the 


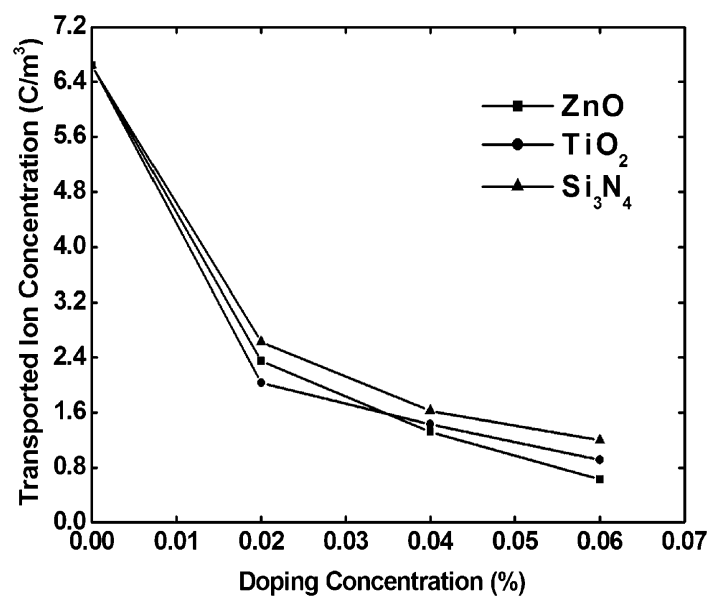

Fig. 3. Transported ion concentration of $\mathrm{ZnO}, \mathrm{TiO}_{2}$, and $\mathrm{Si}_{3} \mathrm{~N}_{4}$ doped LC cells as a function of doping concentrations.

occurrence of the current bump is explained as follows. When a square voltage is applied to the LC cell, the ions in LC host move toward the alignment layers. In the next pulse, the ions will move in the opposite direction since the polarity is changed. Because of the trapping effect at the alignment layers, not all ions will start moving immediately. The total amount of moving ions increases when more and more trapped ions are removed from the alignment layers by the electric field. Finally, some amount of ions reaches the opposite alignment layer and the current starts to decay. The experimental result shows that the transient current bumps are effectively reduced by the addition of different kinds of insulating nanoparticles $\left(\mathrm{ZnO}, \mathrm{TiO}_{2}\right.$, and $\left.\mathrm{Si}_{3} \mathrm{~N}_{4}\right)$. We suggest that the properties of the LC host have been modified by the addition of the guest dopants, presumably due to sufficient ion trapping by the polarized nanoparticles.

By integrating the area below the transient current curve, we can determine the transported ion concentration $n_{t}=$ $1 / A d \int_{0}^{T} I(t) d t$, where $A$ is the pixel area, $d$ is the thickness of the cell, and $T$ is the time at which the charged ions are fully separated. ${ }^{4)}$ The calculated transported ion concentration of different kinds of insulating nanoparticle-doped LC cells with different doping concentration is shown in Fig. 3. We find that the transported ion concentration is decreased apparently when the doping concentration is $0.02 \mathrm{wt} \%$. As the doping concentration increases, the transported ion concentration gradually decreases and tends to reach a minimum value. To investigate the influence of ions on the threshold voltage, the transmittance of the LC cells were measured. For NW configuration, the threshold voltage is defined as the voltage when the light transmittance is $90 \%$ of the initial transmittance at zero voltage. Figure 4 shows the transmittance of doped and undoped TN LC cells, and Fig. 5 shows the corresponding threshold voltages at different doping concentrations. We can see that the $\mathrm{ZnO}, \mathrm{TiO}_{2}$, and $\mathrm{Si}_{3} \mathrm{~N}_{4}$ nanoparticles doped LC cells possess lower threshold voltages compared to that of undoped one. We suggest that this phenomenon was due to the guest dopants whose interaction with ion impurities made the screening effect less effective and allowed the LC molecules to experience a relatively larger effective voltage within the cell and thus lead to the reduction of the threshold voltage. From the

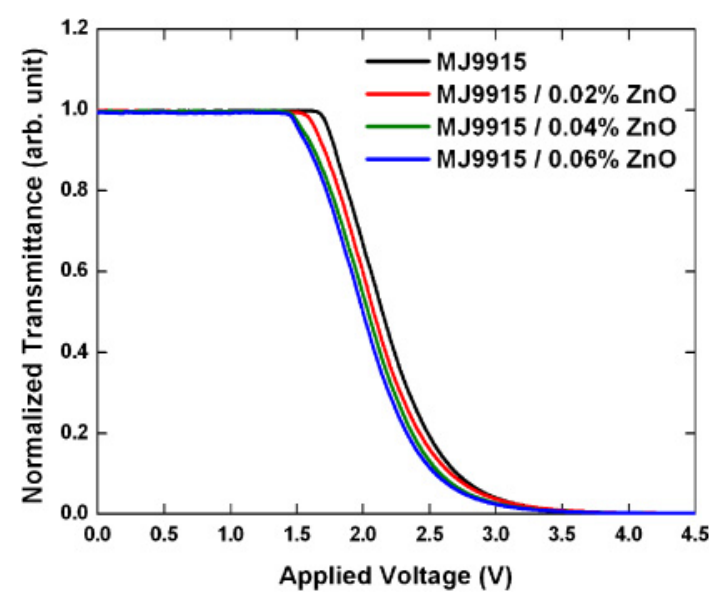

(a)

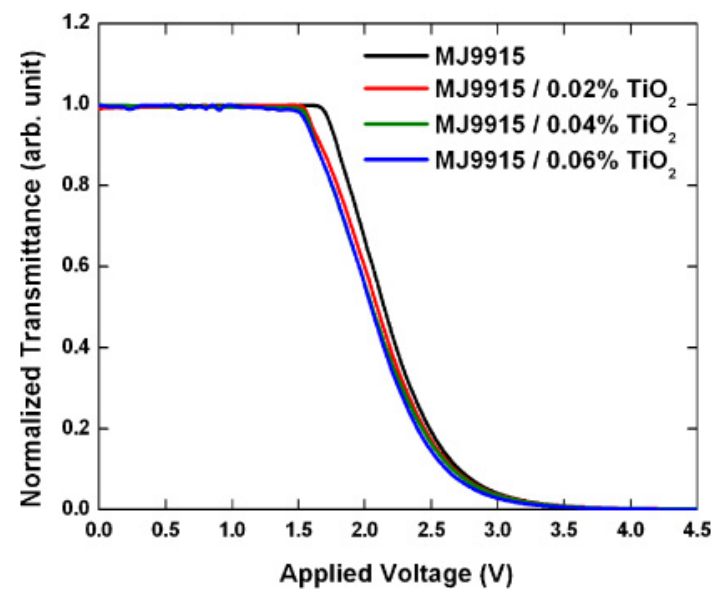

(b)

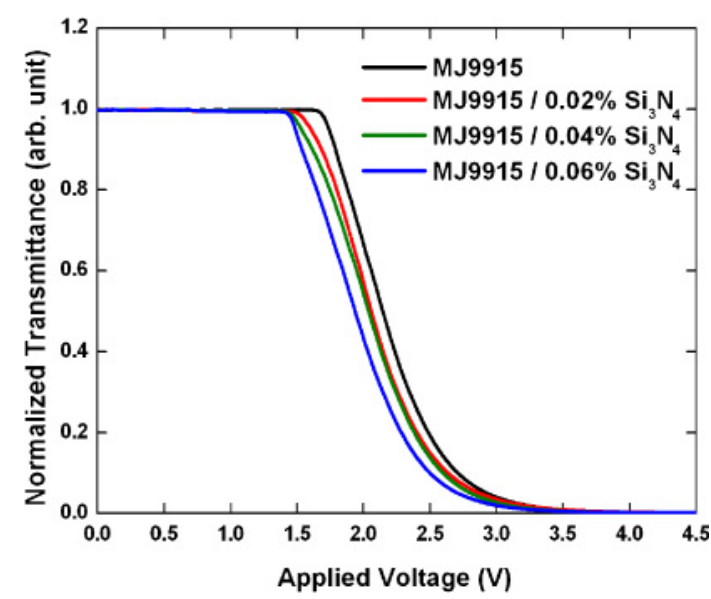

(c)

Fig. 4. (Color online) Measured transmittance of (a) $\mathrm{ZnO}$, (b) $\mathrm{TiO}_{2}$, and (c) $\mathrm{Si}_{3} \mathrm{~N}_{4}$ doped LC cells.

experiments above, we find that the ability to reduce the transported ion concentration and the threshold voltage is apparently not dependent on the type of nanoparticles; this suggests that the ions are physically trapped by the polarized nanoparticles by Coulomb force.

Figures 6(a) and 6(b) are the conceptual depiction of ions moving in the LC cell with and without insulating nanoparticle doping. Figure 6(b) illustrates that the insulating nanoparticles can trap ions in the LC material and leads to 


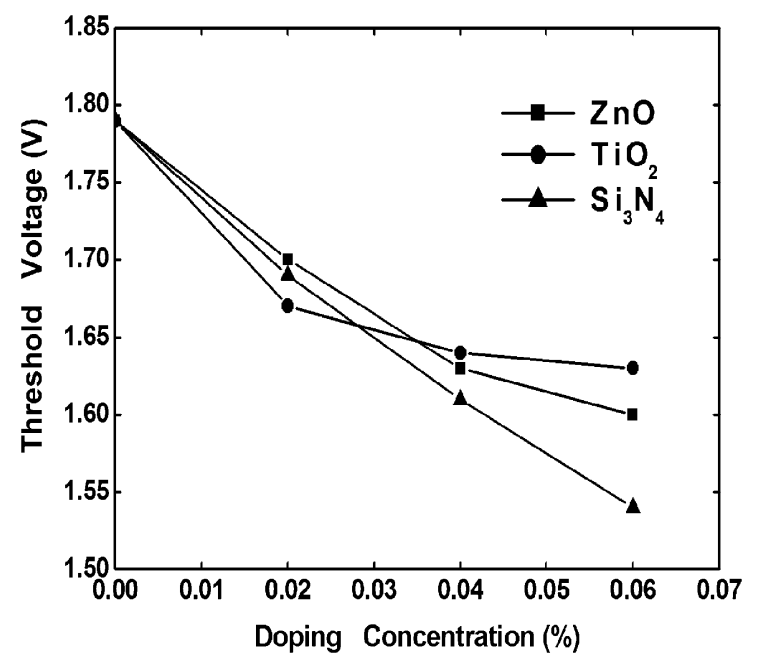

Fig. 5. Threshold voltage of $\mathrm{ZnO}, \mathrm{TiO}_{2}$, and $\mathrm{Si}_{3} \mathrm{~N}_{4}$ doped $\mathrm{LC}$ cells as a function of doping concentrations.

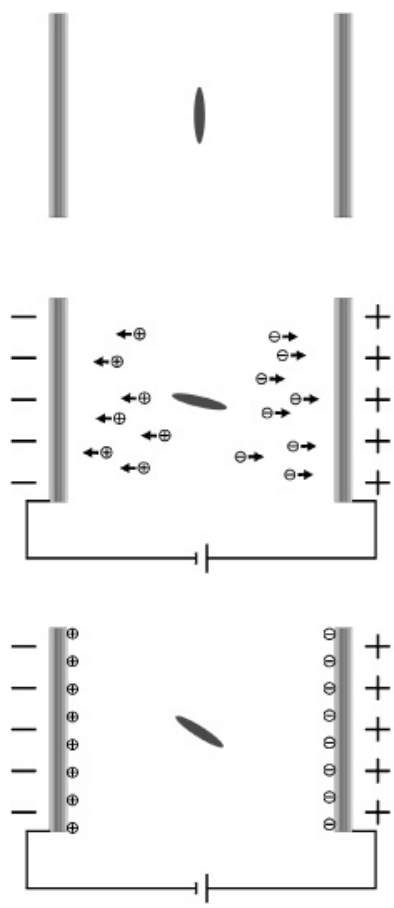

(a)
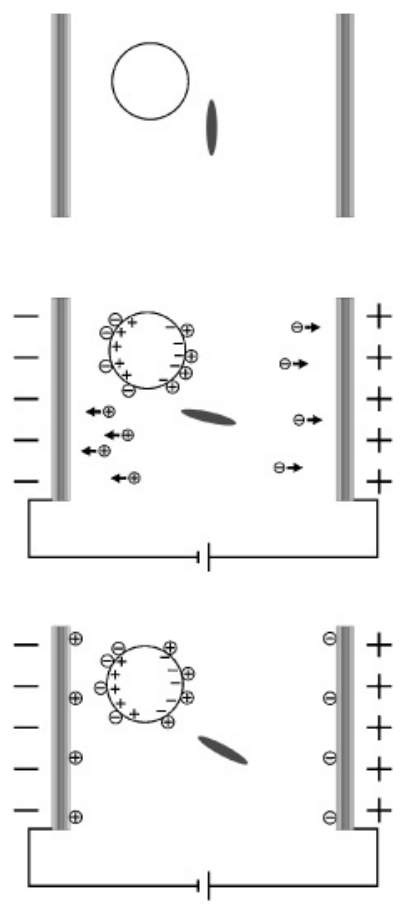

(b)

Fig. 6. Conceptual depiction of ions moving in the cell (a) without and (b) with doping insulating nanoparticles.

fewer ions flowing to two sides of the cell and thus abates the screening effect. For further understanding of the relationship between the reduction of both transported ion concentration and the threshold voltage, we calculated the threshold voltage drop caused by the amount of ions trapped by the nanoparticles. To do the calculation, we treat the LC cell as a capacitor filled by a dielectric material. Since the thickness of the LC cell is very small compared to the length of the cell, we can neglect the fringing effect. The voltage created by the ions that was originally adsorbed on the alignment layers can therefore be calculated by the formula $V=\sigma d / \varepsilon_{0} \kappa$, where $\sigma$ is the surface charge density, $\kappa$ is the dielectric constant of the material filled in the capacitor and $d$ is the thickness of the capacitor. The ions contribute to the

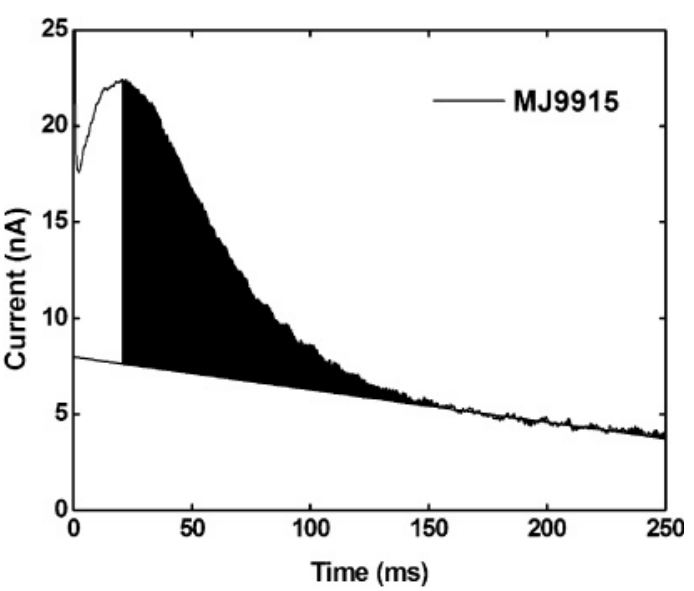

(a)

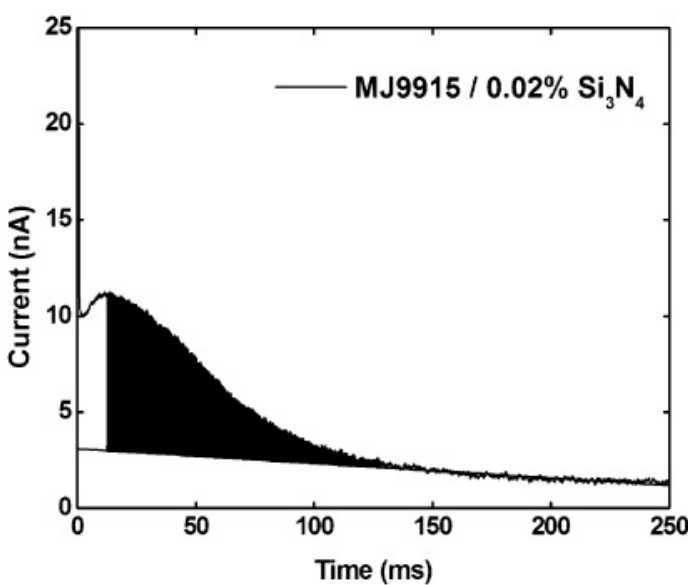

(b)

Fig. 7. Illustration of the integrated area contribute to the surface charge density for (a) undoped and (b) $0.02 \% \mathrm{Si}_{3} \mathrm{~N}_{4}$ doped LC cells.

surface charge density are the integrated area shown in Fig. 7 since the first free ion arrives at the alignment layer when the transient current begins to decay and the decreasing transient current drops to a constant level at the moment when the last free ion reaches the alignment layer. The dielectric constant used in our calculation is the average of the dielectric constant parallel and perpendicular to the LC director where $\kappa_{\|}=12.2$ and $\kappa_{\perp}=4.0$. The thickness of the LC cell is $4 \mu \mathrm{m}$. The calculated threshold voltage drop caused by the difference of surface charge density between the undoped and the $0.02 \% \mathrm{Si}_{3} \mathrm{~N}_{4}$ doped cells is about $0.13 \mathrm{~V}$ which is very close to the measured threshold voltage drop $(0.1 \mathrm{~V})$. This result confirms the relationship between the transported ion concentration and the threshold voltage. The discrepancy between the calculated and the measured voltage drop may come from the slight influence of the guest dopants on the dielectric constant and the elastic constant of LC-nanoparticle dispersion. Figure 8 shows the VHR values of doped and undoped TN LC (MJ9915) cells. In comparison with the CNT-doped cells, ${ }^{14)}$ the insulating nanoparticles doped cells possess a sufficiently high VHR in different doping concentration. Because the nanoparticles are insulating, they will not increase the conductance of LCs. However, the conductance of the CNTs-doped cell will increase when the CNTs are reoriented from planar to 


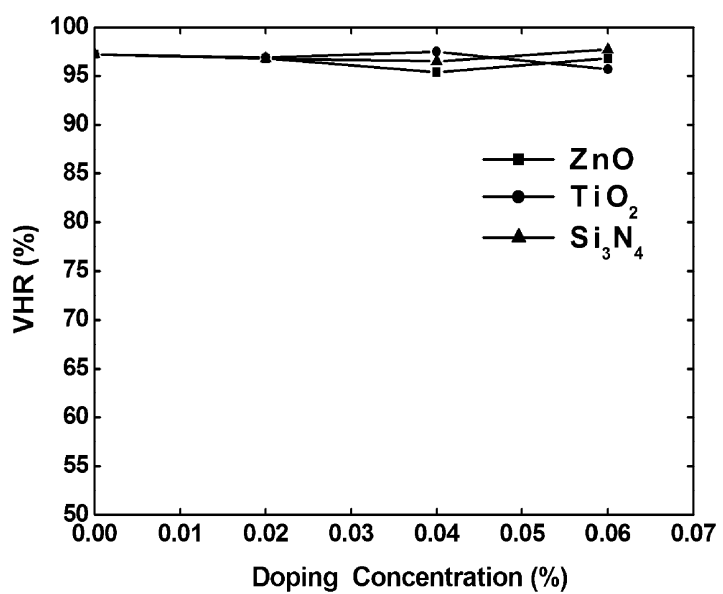

Fig. 8. VHR values of $\mathrm{ZnO}, \mathrm{TiO}_{2}$, and $\mathrm{Si}_{3} \mathrm{~N}_{4}$ doped $\mathrm{LC}$ cells as a function of doping concentrations.

homeotropic orientation by LC molecules above the Freedericksz threshold, ${ }^{15)}$ resulting in a low VHR value.

\section{Conclusions}

In summary, the transient current and the voltage dependent transmittance in TN LC cells doped with $\mathrm{ZnO}, \mathrm{TiO}_{2}$, and $\mathrm{Si}_{3} \mathrm{~N}_{4}$ nanoparticles were studied. The lower transient current found in the doped cells indicates that doping insulating nanoparticles into the LC cell has the ability to decrease the moving-ion density, and thus suppresses the undesired field-screening effect and contributes to a reduction of the threshold voltage. The transmittance curve also confirmed that the screening effect caused by the ion impurity was reduced. The comparison between the calculated and measured threshold voltage drop confirms the relationship between the threshold voltage and the transported ion concentration. We suggest that the ability to reduce the screening effect is due to the ion trapping by insulating nanoparticles which are polarized under an external electric field. Besides, the VHR of the doped cells can still maintain higher than $95 \%$, which indicates that these insulating nanoparticles will not decrease the resistivity of the LC cell. Therefore the insulating nanoparticles are better choices for one to use in order to avoid the disadvantages of LC devices caused by ion impurities.

\section{Acknowledgments}

We thank the Industrial Technology Research Institute for VHR measurements. One of us (C.Y.C.) acknowledges the support from the National Science Council and the Ministry of Education of Taiwan.

1) H. Mada and K. Osajima: J. Appl. Phys. 60 (1986) 3111.

2) C. Colpaert, B. Maximus, and H. Pauwels: Proc. SID Res. Conf., 1993, p. 301.

3) W. Lee, J. S. Gau, and H. Y. Chen: Appl. Phys. B 81 (2005) 171.

4) R. James, G. Stojmenovik, C. Desimpel, S. Vermael, F. A. Fernandez, S. E. Day, and K. Neyts: J. Disp. Technol. 2 (2006) 237.

5) N. Sasaki: Jpn. J. Appl. Phys. 37 (1998) 6065.

6) K. Furuichi, J. Xu, M. Inoue, H. Furuta, N. Yoshida, A. Tounai, Y. Tanaka, A. Mochizuki, and S. Kobayashi: Jpn. J. Appl. Phys. 42 (2003) 4411.

7) W. Lee, C. Y. Wang, and Y. C. Shih: Appl. Phys. Lett. 85 (2004) 513.

8) I. S. Baik, J. Y. Lee, S. Y. Jeon, K. H. An, J. W. Choi, S. H. Lee, and Y. H. Lee: Appl. Phys. Lett. 87 (2005) 263110.

9) T. Miyama, J. Thisayukta, H. Shiraki, Y. Sakai, Y. Shiraishi, N. Toshima, and S. Kobayashi: Jpn. J. Appl. Phys. 43 (2004) 2580.

10) Y. Shiraishi, N. Toshima, K. Maeda, H. Yoshikawa, J. Xu, and S. Kobayashi: Appl. Phys. Lett. 81 (2002) 2845.

11) F. Haraguchi, K. I. Inoue, N. Toshima, S. Kobayashi, and K. Takatoh: Jpn. J. Appl. Phys. 46 (2007) 796.

12) Y. Reznikov, O. Buchnev, O. Tereshchenko, V. Reshetnyak, A. Glushchenko, and J. West: Appl. Phys. Lett. 82 (2003) 1917.

13) A. Glushchenko, C. II Cheon, J. West, F. Li, E. Büyüktanir, Y. Reznikov, and A. Buchnev: Mol. Cryst. Liq. Cryst. 453 (2006) 227.

14) P. S. Chen, C. C. Huang, Y. W. Liu, and C. Y. Chao: Appl. Phys. Lett. 90 (2007) 211111

15) I. Dierking, G. Scalia, P. Morales, and D. LeClere: Adv. Mater. 16 (2004) 865 .

16) C. Colpaert, B. Maximus, and A. D. Meyere: Liq. Cryst. 21 (1996) 133. 